\title{
BMJ Open Detailed systematic analysis of recruitment strategies in randomised controlled trials in patients with an unscheduled admission to hospital
}

Ceri Rowlands, ${ }^{1}$ Leila Rooshenas, ${ }^{1,2}$ Katherine Fairhurst, ${ }^{1,2}$ Jonathan Rees, ${ }^{2,3}$ Carrol Gamble, ${ }^{4}$ Jane M Blazeby ${ }^{1,2,3}$

To cite: Rowlands C, Rooshenas L, Fairhurst K, et al. Detailed systematic analysis of recruitment strategies in randomised controlled trials in patients with an unscheduled admission to hospital. BMJ Open 2018;8:e018581. doi:10.1136/ bmjopen-2017-018581

- Prepublication history for this paper is available online. To view these files, please visit the journal online (http://dx.doi. org/10.1136/bmjopen-2017018581).

Received 11 July 2017 Revised 5 December 2017 Accepted 8 December 2017

Check for updates

${ }^{1}$ MRC ConDuCT-II Hub for Trials Methodology Research, School of Social and Community Medicine, University of Bristol, Bristol, UK

${ }^{2}$ School of Population Health

Sciences, Bristol Medical School, University of Bristol, Bristol

${ }^{3}$ Division of Surgery, Head and Neck, University Hospitals Bristol NHS Foundation Trust, Bristol, UK

${ }^{4}$ MRC North West Hub for Trials Methodology Research, Institute of Translational Medicine,

University of Liverpool,

Liverpool, UK

Correspondence to

Dr Leila Rooshenas;

Leila.rooshenas@bristol.ac.uk

\section{ABSTRACT}

Objectives To examine the design and findings of recruitment studies in randomised controlled trials (RCTs) involving patients with an unscheduled hospital admission (UHA), to consider how to optimise recruitment in future RCTs of this nature.

Design Studies within the ORRCA database (Online Resource for Recruitment Research in Clinical TriAls; www.orrca.org.uk) that reported on recruitment to RCTs involving UHAs in patients $>18$ years were included. Extracted data included trial clinical details, and the rationale and main findings of the recruitment study. Results Of 3114 articles populating ORRCA, 39 recruitment studies were eligible, focusing on 68 real and 13 hypothetical host RCTs. Four studies were prospectively planned investigations of recruitment interventions, one of which was a nested RCT. Most recruitment papers were reports of recruitment experiences from one or more 'real' RCTs $(n=24)$ or studies using hypothetical RCTs $(n=11)$. Rationales for conducting recruitment studies included limited time for informed consent (IC) and patients being too unwell to provide IC. Methods to optimise recruitment included providing patients with trial information in the prehospital setting, technology to allow recruiters to cover multiple sites, screening logs to uncover recruitment barriers, and verbal rather than written information and consent.

Conclusion There is a paucity of high-quality research into recruitment in RCTs involving UHAs with only one nested randomised study evaluating a recruitment intervention. Among the remaining studies, methods to optimise recruitment focused on how to improve information provision in the prehospital setting and use of screening logs. Future research in this setting should focus on the prospective evaluation of the well-developed interventions to optimise recruitment.

\section{INTRODUCTION}

Recruitment to randomised controlled trials (RCTs) is the biggest obstacle to successful trial conduct. ${ }^{1}$ Recruitment may be particularly challenging among patients who have an unscheduled hospital admission (UHA). In this situation, the clinical condition of

\section{Strengths and limitations of this study}

- This review is the first to focus on the complex issue of recruitment to RCTs involving patients undergoing an unscheduled hospital admission (UHA).

- This review is the first publication to use the ORRCA database (Online Resource for Recruitment Research in Clinical TriAls) in generating recruitment research.

- The ORRCA database continues to evolve as updates encompass newly published recruitment research. Updates to the database may have generated further UHA research articles since the completion of this review.

the potential participant and the demanding working environment can complicate the process of identifying eligible patients, approaching them, and obtaining informed consent for trial participation. Patients are often in pain, unwell, and anxious about the underlying problem. There may also be time limitations due to the urgent need to deliver the clinical treatments under evaluation. ${ }^{23}$

There are a number of existing systematic reviews of methods to optimise recruitment to trials in a variety of clinical contexts and patient populations, including cancer, ${ }^{4-6}$ primary care $^{7}$ geriatrics $^{8} 9$ and minority community patients, ${ }^{10}$ or a mix of clinical settings. ${ }^{11-17}$ None specifically focus on recruitment of patients undergoing UHA.

Established methods exist for recruiting potential RCT participants who are unwell or unconscious and lack capacity. In these circumstances, permission for enrolment into an RCT may be sought from a surrogate decision-maker $(\mathrm{SDM}),{ }^{18}$ or through deferred consent (also known as 'exception from informed consent'), a process where a participant is recruited into the trial in order for urgent treatment to be provided 
and subsequently asked to provide written consent for ongoing participation once they regain capacity. ${ }^{19}$ However, recruitment may be particularly challenging where patients are acutely unwell, but retain capacity to decide on enrolment into an RCT. Reviewing the literature on how to optimise recruitment in this setting may lead to valuable insights and identify areas where further research is needed.

The aim of this paper was to examine the design and findings of recruitment studies in RCTs involving patients with a UHA, to consider how to optimise recruitment in future RCTs of this nature.

\section{METHODS}

\section{Search strategy}

Articles were identified through manually screening each entry within the ORRCA recruitment research database (Online Resource for Recruitment research in Clinical triAls; http://www.orrca.org.uk/). The ORRCA project was funded by the UK Medical Research Council Hubs for Trials Methodology Research Network. It provides a comprehensive online database of published empirical and non-empirical papers about recruitment to clinical research. ORRCA is populated from an extensive systematic search of the Cochrane Library, MEDLINE (Ovid), SCOPUS, ERIC and SCI-EXPANDED and SSCI (via ISI Web of Science). The search strategy employed by ORRCA was based on a Cochrane systematic review of trial recruitment. ${ }^{16}$ Further details about the formation of the ORRCA database is reported on their website (http:// www.orrca.org.uk/). In this review, a full up-to-date copy of the ORRCA database was obtained in January 2016, and the database was searched in February 2016. At this stage, ORRCA contained publications relevant to recruitment published up to and including the end of December 2014. Publications from 2015 onwards were not available due to ongoing work in processing articles in the ORRCA database.

\section{Study eligibility criteria}

Any study held within ORRCA that reported on recruitment to RCTs involving UHAs was eligible for inclusion. UHA was defined as an unscheduled admission to hospital at short notice because of clinical need. This included prehospital care, intensive care unit admissions, and accident and emergency (A\&E) department attendances. Studies that reported on a mix of patients undergoing scheduled and unscheduled admissions were eligible if the findings for the UHA study population were described separately. Papers that reported recruitment strategies for a mixture of RCTs and other types of research (ie, non-RCTs) were only eligible for inclusion if the recruitment strategies for RCTs were described separately. RCTs that involved children (age $<18$ years) or patients with acute psychiatric illness were excluded, as these patients would not normally be subject to the usual recruitment processes due to differences in the consent processes. Systematic reviews of methods to optimise recruitment were scrutinised for relevant primary articles, but not included in the analysis. Editorials and studies of recruitment to non-RCTs were excluded. Abstracts were also excluded because these rarely included the necessary contextual information and data needed to make a meaningful contribution to the dataset for this study.

\section{Screening and selection process}

One author (CR) screened all articles included within the ORRCA database. Duplicate screening was carried out by one other author (KF) on $10 \%$ of the database. KF was blinded to the original screening decisions. Papers were assessed at title and abstract level according to the eligibility criteria. Differences in opinion were resolved by discussion between $\mathrm{CR}$ and $\mathrm{KF}$, and any remaining differences in opinion were referred to another member of the study team (JMB) if required. The aim was to reach agreement for screening decisions on all studies within this sample. Agreement was reached on 271/300 articles screened. Of the 29 discrepancies raised, 22 were resolved following discussion between CR and KF. The seven remaining papers were discussed with a third author (JMB), which resulted in two of these papers being included and five being excluded. No paper which was suggested to be included by the second reviewer was eventually included in the review.

We calculated a kappa statistic for the double screened articles above. Ten per cent of articles were double screened with a Kappa $=0.677 \quad$ (SE 0.048, P<0.001) suggesting 'good' agreement. As such, the remaining $90 \%$ of articles in the ORRCA database were screened by a single screener (CR). The third arbiter (JMB) involved in screening the $10 \%$ sample was also consulted for screening the remaining $90 \%$ of articles in the ORRCA database, in instances where the single screener (CR) was uncertain about an article's eligibility.

\section{Definitions: host RCT}

All studies focused on recruitment to one or more specific 'host' RCTs. In this paper, a host RCT refers to the underlying randomised controlled trial (ie, addressing a clinical question) in which the recruitment of participants took place. A host RCT could be a pilot or a main trial. Some recruitment papers reported on community consultations in which the views of the public were sought to establish the likelihood of recruitment success or acceptability of a proposed trial. This approach is typically used when the study population may be critically ill at the time of recruitment (and therefore may be unable to provide full, written informed consent).$^{20}$ In recognition of this, a clear differentiation was made between studies that focused on recruitment to an existing clinical RCT (a 'real' host RCT) versus potential recruitment to an RCT that did not exist (a 'hypothetical' host RCT), but is proposed to exist in order to estimate its acceptability to potential participants. A 'recruitment study' refers to 
Table 1 Number of included studies, host RCTs and hypothetical RCTs categorised according to the new recruitment study types (A to D)

\begin{tabular}{|c|c|c|c|c|}
\hline Category & Recruitment study design & $\begin{array}{l}\text { Recruitment studies } \\
(\mathrm{n}=39)\end{array}$ & $\begin{array}{l}\text { Real host RCTs } \\
(\mathrm{n}=68)\end{array}$ & $\begin{array}{l}\text { Hypothetical host RCTs } \\
(n=13)\end{array}$ \\
\hline$A$ & $\begin{array}{l}\text { RCTs of interventions to optimise recruitment } \\
\text { nested within one or more host RCTs }\end{array}$ & 1 & 1 & NA \\
\hline$B$ & $\begin{array}{l}\text { Prospectively designed, non-randomised } \\
\text { studies of interventions to optimise } \\
\text { recruitment to one or more host RCTs }\end{array}$ & 3 & 3 & NA \\
\hline $\mathrm{D}$ & $\begin{array}{l}\text { Studies to consider recruitment within } \\
\text { proposed hypothetical RCTs (commonly } \\
\text { known as community consultations) }\end{array}$ & 11 & NA & $13+$ \\
\hline
\end{tabular}

${ }^{\star}$ The 24 recruitment studies reported data from 64 real host RCTs, that is, a number of recruitment studies reported data from more than one real host RCT.

†The 11 recruitment studies reported data from 13 hypothetical studies, that is, two recruitment studies reported data from more than one hypothetical host RCT.

NA, not applicable-category does not apply to type of host RCT; RCT, randomised controlled trial.

research into the process of recruiting eligible participants, in the context of one or more real or hypothetical host RCTs.

\section{Definitions: recruitment study design}

In order to group similarly designed recruitment studies together and enhance data analysis, a new categorisation system for different recruitment study designs was developed (Categories A to D). Consideration was given to the design of the recruitment study and whether a real or hypothetical host RCT was used. The categories are provided in table 1 .

\section{Contacting study authors}

If an appropriate recruitment study did not adequately describe the host trial, the study authors were contacted by email to determine whether the host trial met the eligibility criteria. Three attempts, each 2 weeks apart, were made to contact study authors. If no response was received, then the paper was excluded.

\section{Data extraction and synthesis}

CR extracted the data using a predesigned and piloted data extraction form. Data extracted from eligible studies included clinical details of the underlying host RCT, the rationale for conducting a recruitment study, a summary of the recruitment study findings, recommendations for improving recruitment and suggestions for further research. Where specific recruitment interventions had been evaluated, further details regarding the interventions were collected, including the timing of information exchange, informed consent and randomisation. No statistical analyses were planned, as the review was expected to provide a descriptive analysis of results due to the anticipated heterogeneous nature of recruitment strategies presented. During the process, multiple meetings were undertaken with JMB and LR to examine papers and check data extraction processes as required.

\section{RESULTS}

\section{Study selection}

A total of 3114 articles were identified within the ORRCA database. After initial screening at title and abstract level, 3044 articles were excluded, leaving 70 potentially eligible for which full texts were obtained. A further 31 articles were excluded following full-text screening. Duplicate screening did not produce any discrepancies that could not be resolved through discussion. In total, 39 recruitment studies ${ }^{21-58}$ were identified, which reported results from 68 real host RCTs and 13 hypothetical host RCTs and were included in this review.

A number of recruitment studies described results obtained from more than one real or hypothetical RCT (figure 1).

The 68 real host trials (around which recruitment was focused) were predominantly multicentre RCTs (63/68) with large study populations (median, 624 participants; range, 4-58 050) and typically evaluated non-invasive medical interventions $(61 / 68)$ (table 2). The apparent predominance of RCTs in neurology is caused by one recruitment study that included data from 32 separate RCTs. With exception to this, the clinical settings of the host RCTs varied, encompassing several medical disciplines.

The majority of recruitment studies were simple descriptive non-randomised studies reporting previous experiences/challenges of recruitment to a host RCT (Category $\mathrm{C}, \mathrm{n}=24)$. There were 11 that proposed a hypothetical RCT to a study population (Category $\mathrm{D}, \mathrm{n}=11$ ). Only one article used what is considered to be the optimal method for evaluating an intervention-a randomised comparison of a recruitment intervention nested within a host RCT (Category A). Three studies prospectively evaluated recruitment interventions using non-randomised study designs (Category B, table 1). 


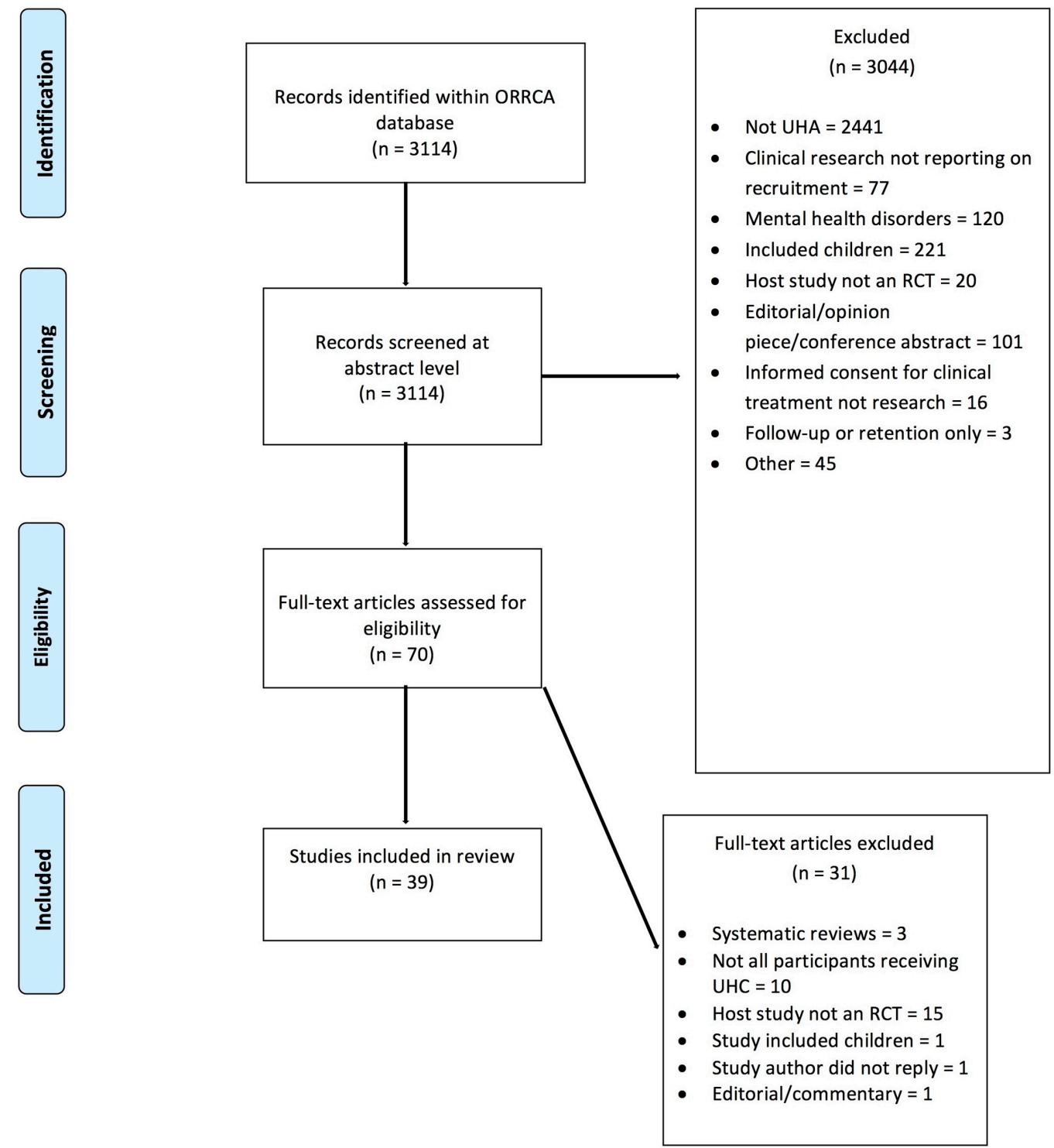

Figure 1 Study selection PRISMA flow diagram. ORRCA, Online Resource for Recruitment Research in Clinical TriAls; PRISMA, Preferred Reporting Items for Systematic Review and Meta-Analysis; RCT, randomised controlled trial; UHA, unscheduled hospital admission; UHC, unplanned hospital care.

\section{RCTs of interventions to optimise recruitment nested within one or more host RCTs (Category A)}

Only one of the included recruitment studies investigated two recruitment strategies using a randomised design (Category A studies, table 3). The rationale for this study was the limited time available for recruitment due to the acute medical treatment required by patients. ${ }^{21}$ Patients randomised to the intervention group received 'advanced notification' of the trial (via fax or phone) designed to offer patients more time to consider trial participation, compared with the control group who only received information once they met with the clinical team. Consent to participate in the host RCT was obtained in 27/50 (54\%) and 25/50 $(50 \%)$ patients in the intervention and control groups, respectively $(\mathrm{P}=0.69)$. Although no improvement in overall recruitment rates was demonstrated using advance notification, the provision of early information was demonstrated to be feasible.

\section{Prospectively designed, non-randomised studies of} interventions to optimise recruitment to one or more host RCTs (Category B)

The common rationale for this type of recruitment study design (Category B studies, table 3) was the limitations of time when dealing with patients who required acute treatment. The need for urgent treatment was thought to hinder the ability of the study team to gain informed consent (IC) for trial participation.

The strategy of optimising information provision in the prehospital setting used in the one Category A study was mirrored by two Category B studies, which used the presence of prehospital staff to engage potential trial participants. ${ }^{22}{ }^{24}$ Recruitment in these studies was reportedly 
Table 2 Summary characteristics of the host RCTs and hypothetical host RCTs in this review

\begin{tabular}{|llll|}
\hline Trial characteristic & $\begin{array}{l}\text { Real RCTs } \\
(\mathbf{n = 6 8 )}\end{array}$ & $\begin{array}{l}\text { Hypothetical } \\
\text { RCTs } \\
(\mathbf{n}=\mathbf{1 3})\end{array}$ & $\begin{array}{l}\text { Total } \\
(\mathbf{n}=\mathbf{8 1})\end{array}$ \\
\hline Clinical setting & & & \\
\hline Neurology & $39^{*}$ & 4 & 43 \\
\hline Cardiology & 8 & 4 & 12 \\
\hline Obstetrics & 3 & 1 & 4 \\
\hline Infection & 3 & 0 & 3 \\
\hline Trauma & 6 & 3 & 9 \\
\hline Critical care & 9 & 1 & 10 \\
\hline
\end{tabular}

Type of interventions

\begin{tabular}{|c|c|c|c|}
\hline Invasive/non-invasive & $5 / 61$ & $4 / 9$ & $9 / 70$ \\
\hline Unknown & 2 & 0 & 2 \\
\hline \multicolumn{4}{|l|}{ Trial design } \\
\hline Main RCT/pilot RCT & $65 / 3$ & $\mathrm{n} / \mathrm{a}$ & $65 / 3$ \\
\hline $\begin{array}{l}\text { Two groups/more than } \\
\text { two groups }\end{array}$ & $63 / 5$ & $11 / 2$ & $74 / 7$ \\
\hline \multicolumn{4}{|l|}{ No of centres $†$} \\
\hline $\begin{array}{l}\text { Single centre/ } \\
\text { multicentre/unknown }\end{array}$ & $3 / 63 / 2$ & $0 / 4 / 9$ & $3 / 67 / 11$ \\
\hline$<20$ centres & 18 & & \\
\hline 20-100 centres & 33 & & \\
\hline$>100$ centres & 15 & & \\
\hline $\begin{array}{l}\text { Median no of centres } \\
\text { (range) }\end{array}$ & $\begin{array}{l}45 \\
(1-818)\end{array}$ & & \\
\hline \multicolumn{4}{|l|}{ No of participants $†$} \\
\hline$<500$ & 22 & & \\
\hline $500-1500$ & 32 & & \\
\hline$>1500$ & 14 & & \\
\hline $\begin{array}{l}\text { Median no of } \\
\text { participants (range) }\end{array}$ & $\begin{array}{l}624 \\
(4-58050)\end{array}$ & & \\
\hline
\end{tabular}

*One recruitment paper included data from 32 stroke host RCTs.

†Hypothetical RCT data did not include information beyond single or multicentre, nor the suggested number of participants.

$\mathrm{RCT}$, randomised controlled trial.

optimised through provision of brief verbal information to participants as they travelled to hospital, and initial verbal consent that sought permission to deliver the emergency trial intervention. Further information was provided and written consent was subsequently obtained when the patients were stabilised, in hospital. One of these studies ${ }^{24}$ also provided training to prehospital staff to improve their understanding of trial conduct, and devised a simple assessment of capacity to ensure that patients' initial verbal consent was valid.

The remaining prospectively designed (Category B) study focused on using technology to enhance recruitment during an influenza outbreak. ${ }^{23}$ As patient numbers would be expected to rise rapidly across a wide geographical area, the study team devised a system which provided them with automated, real-time alerts whenever an eligible participant was identified in each hospital. This allowed one centralised study team to cover numerous study sites, enhancing recruitment opportunities. Although none of these studies provided numerical evidence of the effectiveness of their recruitment strategies, all authors concluded that their presented strategies were feasible and acceptable for use in UHA RCTs.

\section{Studies describing recruitment experiences involving one or more host RCTs (Category C)}

Rationales for reporting authors' experiences of recruitment were similar to those in prospectively designed studies, including the limited time available for consent $(\mathrm{n}=13)$, and recruitment difficulties caused by the clinical condition of the patients $(n=18)$ (Category C studies, table 4). Some studies were prompted by a host trial encountering recruitment difficulties $(n=3)$. Of the 24 non-randomised studies describing recruitment experiences involving one or more host RCTs, 14 were observational and five were qualitative studies.

These studies reported experiences of trial participants and SDMs, or extracted verbatim information from written patient information sheets. Recommendations for optimising recruitment also mirrored Category A and B studies, highlighting the acceptability of verbal information provision and consent, or deferring consent altogether until an unwell patient is suitably stabilised. Additional benefits were seen in RCTs that used data from screening logs to uncover recruitment barriers and trials that performed regular site visits. ${ }^{29-31} 3335383944$ A subsection of qualitative studies, although not presenting recommendations for future trial conduct, highlighted their findings that many patients or SDMs who had provided consent to participate in an RCT did not recall much of the information provided to them during the consent process, suggesting that work was needed to improve consent in this setting. ${ }^{31} 3240$

\section{Studies to consider recruitment within proposed hypothetical RCTs (commonly known as community consultations) (Category D)}

Eleven studies reported community views about proposed 'hypothetical' RCTs (Category D studies, table 5). Although the rationales for conducting the studies were similar to studies involving real host RCTs (unwell patients and lack of time for consent), the study designs were varied including questionnaire surveys, interviews and focus group meetings. Verbal information provision, verbal consent and recruitment in the prehospital setting were identified as helpful recruitment strategies in these studies. However, these articles raised new issues around the appropriateness of using of SDMs when patients are too unwell to provide consent for themselves and raised additional issues around who the SDM should be (next of kin (NOK) or an available physician). Some studies found a preference for the use of SDMs, ${ }^{50} 5256$ while others expressed that NOK or physicians should not be used for their specific hypothetical RCTs. ${ }^{53558}$ 


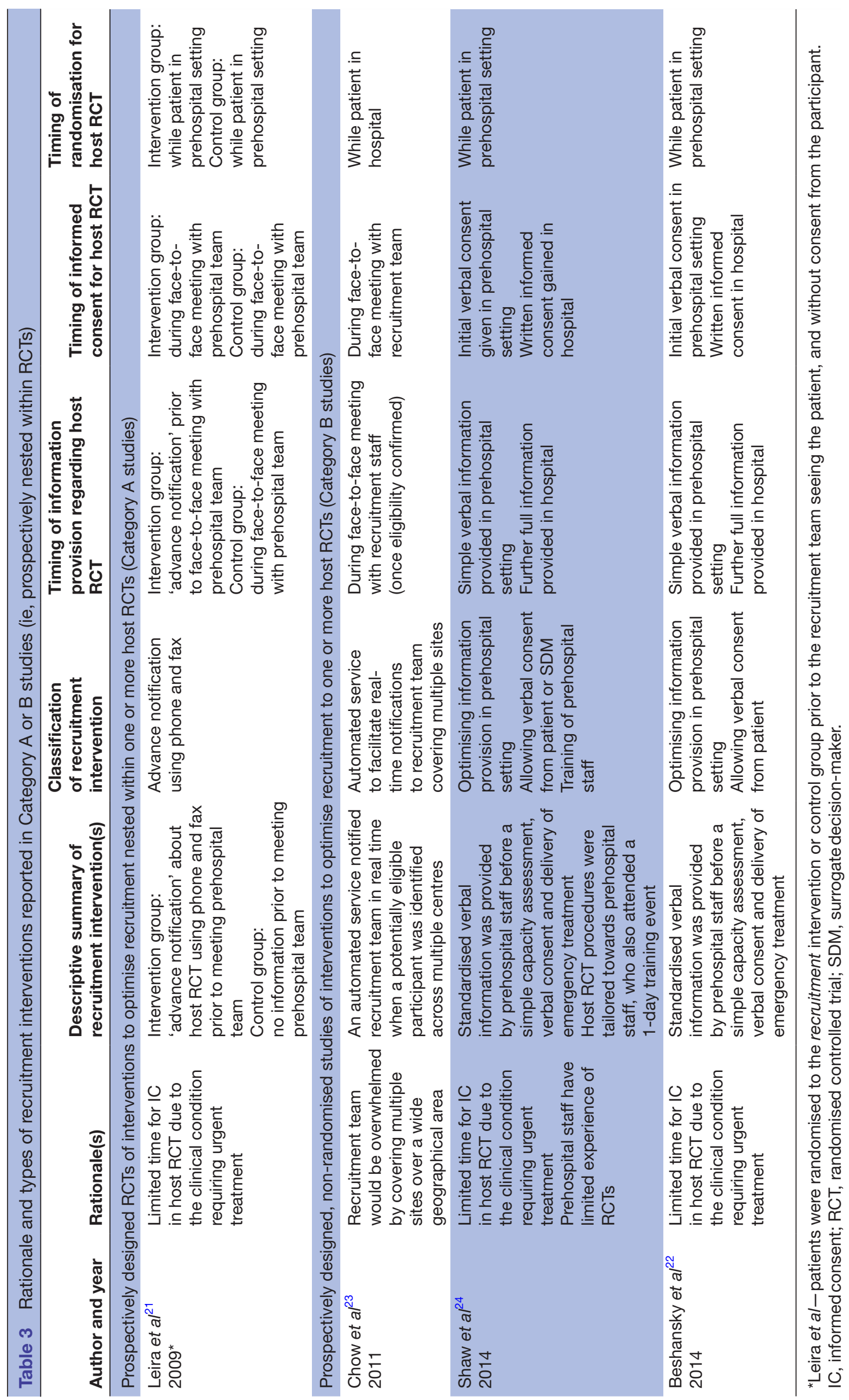


Table 4 Frequency of rationales, study designs and recommendations from non-randomised studies describing recruitment experiences involving one or more host RCTs (ie, Category C studies)

\section{Recruitment study characteristic}

Rationale

\section{Description}

Patients too unwell to provide IC

Limited time for IC in host RCT due to the clinical condition requiring urgent treatment

Host RCT not meeting recruitment targets (at one or more sites) or terminated due to poor recruitment

To better understand the impact of altering eligibility criteria on recruitment

To better understand the impact of availability of SDMs on recruitment

To better understand the recruitment process in a host RCT

To better understand clinicians reasons for refusing patient participation in host RCT

\section{Recruitment study design}

Observational study of recruitment

Qualitative studies of host participants/SDMs or PIS

Survey of host RCT participants

Survey of clinical staff involved in host RCT

Simulation study evaluating the effect of altering eligibility criteria in multiple host RCTs

Meta-analysis of recruitment data in host RCTs

To provide RCT information verbally and allow a verbal consent
process

\section{optimising recruitment} in future RCTs or areas for further research into recruitment†

To use a screening log can to provide insight into recruitment difficulties

$\begin{array}{lr}\text { Patients or SDM were unable to recall key RCT information after } & 5 \\ \text { providing IC† } & 4 \\ \text { To use a 'waiver of consent'/'deferred consent'/‘EFIC' } & 3 \\ \text { To perform regular site visits } & 2 \\ \text { To use a broad eligibility criteria/broad therapeutic window } & 2 \\ \text { To use SDMs } & 2 \\ \text { Novel methods for obtaining IC are required† } & 1 \\ \text { To replace poorly recruiting centres } & 1 \\ \text { To approach more eligible patients } & 1 \\ \text { To survey staff involved with host RCT to provide insight into } & \\ \text { recruitment difficulties } & \end{array}$

*Each study may appear more than once in the relevant characteristics section (eg, if it described $>1$ rationale or produced $>1$ finding/ recommendation).

†ltems for further research and not recommendations for optimising recruitment.

EFIC, exception from informed consent; IC, informed consent; PIS, patient information sheet; RCT, randomised controlled trial; SDM, surrogate decision-maker.

\section{DISCUSSION}

This review aimed to examine and summarise studies and methods used to optimise recruitment in RCTs in patients with an UHA. It had the purpose of using the information to consider how to optimise recruitment in this challenging clinical setting in future studies. In the ORCCA database of recruitment research, only 39 out of a possible 3114 articles (1.25\%) focused on recruitment to RCTs in the UHA setting. Only one of these studies was a randomised comparison of recruitment strategies; the majority of studies consisted of simple study designs describing recruitment experiences. Eleven further studies involved hypothetical RCTs, and while of some value, it is uncertain how these types of investigations translate into optimal RCT design. This work therefore highlights the need for development of interventions to optimise recruitment in the UHA setting and prospective evaluation of their effectiveness and acceptability. 
Table 5 Frequency of rationales, study designs, main findings and recommendations from non-randomised studies designed study to consider recruitment within proposed hypothetical RCTs (ie, Category D studies, commonly known as community consultations)

\begin{tabular}{|c|c|c|}
\hline Recruitment study characteristic & Description & $\begin{array}{l}\text { D recruitment } \\
\text { studies }(n=11)^{*}\end{array}$ \\
\hline \multirow[t]{2}{*}{ Rationale } & Patients too unwell to provide IC & 9 \\
\hline & $\begin{array}{l}\text { To explore the accuracy of decisions made by NOK when } \\
\text { acting as SDM }\end{array}$ & 1 \\
\hline \multirow{3}{*}{ Recruitment study design } & Face-to-face interview & 4 \\
\hline & Telephone survey & 1 \\
\hline & Focus group meetings & 1 \\
\hline \multirow{7}{*}{$\begin{array}{l}\text { Recommendations for optimising recruitment in } \\
\text { future RCTs or main findingst }\end{array}$} & To use a physician as a SDM & 4 \\
\hline & $\begin{array}{l}\text { To provide RCT information verbally and allow a verbal } \\
\text { consent process }\end{array}$ & 1 \\
\hline & To allow recruitment in prehospital setting & 1 \\
\hline & $\begin{array}{l}\text { To perform community consultations to estimate host } \\
\text { RCT recruitment ratest }\end{array}$ & 1 \\
\hline & Not to use a physician as a SDM & 1 \\
\hline & Not to use EFIC & 1 \\
\hline & $\begin{array}{l}\text { To perform community consultations to aid selection of } \\
\text { relevant study outcomes } \dagger\end{array}$ & 1 \\
\hline
\end{tabular}

*Each study may appear more than once in the relevant characteristics section (eg, if it described $>1$ rationale or produced $>1$ finding/ recommendation).

†ltems reported as main findings, but not recommendations for optimising recruitment.

EFIC, exception from informed consent; IC, informed consent; NOK, next of kin; RCT, randomised controlled trial; SDM, surrogate decision-maker.

\section{Comparison to existing literature}

There have been several reviews that have summarised the evidence for optimising recruitment in trials in other clinical conditions or contexts, including cancer, ${ }^{4-6}$ geriatrics, ${ }^{8} 9$ primary care $^{7}$ and a mix of clinical settings. ${ }^{11-17}$ Similar to our findings, these reviews have commonly highlighted the lack of highquality evaluations of recruitment interventions. ${ }^{6} 16$ Despite this, some of the reviews have identified effective recruitment strategies, although these vary in the extent to which they are likely to be transferrable to the UHA setting. For example, it is unclear if interventions such as telephone reminders, ${ }^{16}{ }^{17}$ 'education sessions' about the health condition ${ }^{11}$ and use of monetary incentives ${ }^{11} 17$ are as effective or appropriate in the UHA context, given the specific factors that may compromise recruitment in this setting (eg, patients in pain/distress, short timeframes for recruitment, busy settings etc). Other reviews have drawn attention to interventions aimed at recruiters, such as appropriate training/guidance, ${ }^{12}$ reduction of clinical workload ${ }^{712}$ and 'research protected' time. ${ }^{12}$ These have potential to be helpful in trials conducted in the UHA setting, although further research is needed to examine the content/nature of the training materials needed and the logistics of implementing these types of interventions. Finally, some reviews have shown that features of RCT study design-such as open rather than placebo-controlled trials-are associated with better recruitment outcomes. ${ }^{16}{ }^{17}$ Recommendations that advise against particular study designs may limit the quality of evidence generated to guide future patient care and dissuade from the most important (and appropriate) clinical questions from being addressed. Furthermore, there is a growing body of evidence to indicate that it is possible to recruit to more complex RCTs with appropriate training and support. ${ }^{59}$ More generally, use of integrated qualitative research to understand and address recruitment difficulties is being increasingly recommended in more recent reviews ${ }^{12} 60$ and is likely to inform novel insights if applied to trials in UHA settings. 
Some reviews and individual studies have produced recommendations that are likely to be particularly relevant for developing UHA-specific recruitment strategies for future evaluation. These strategies may tackle some of the context-specific difficulties that are likely to be experienced in UHA settings. For example, one systematic review focusing on recruitment to RCTs involving patients with cancer or organ failure highlighted the potential for providing audiovisual information (such as a video to explain the RCT) to facilitate RCT recruitment. ${ }^{59}$ Such an approach may be helpful in the UHA setting, given that large amounts of written information may not be appropriate in patients who are in pain or feeling distressed. This recommendation is also in keeping with guidance issued by the National Health Service Health Research Authority, which suggests using alternatives to written information. ${ }^{61}$

A verbal exchange of information and providing initial verbal consent has been previously suggested as a preferred strategy to written alternatives in RCTs taking place in the emergency setting. ${ }^{62}$ It is agreed that for UHAs, this could be preferable, although it is considered that further work is needed to develop this type of verbal consent to ensure that quality assurance is still achieved for consent. Another suggested solution to this problem is the use of an independent patient advocate, who may oversee such conversations between trial team members and acutely unwell patients, to verify that appropriate information exchange took place and to act as an assessor of a patient's willingness to participate in the RCT. ${ }^{63}$ This strategy provides one potential solution to a significant obstacle in recruitment of patients undergoing an UHA. However, this may be practically difficult to achieve given that UHAs can occur at any time of the day or night and such trained patient advocates would also need to be available during these times, making a trial more expensive.

Finally, a non-systematic review article focusing on recruitment to emergency medicine research also highlighted a similar problem in a lack of high-quality evidence on recruitment. It suggested support for the use of deferred consent (also known as exception from informed consent), which was highlighted in this review and additionally raised the issue that the use of SDMs for consent may be problematic due to the pressures of time and the emotional stress family members will be under while a relative is acutely unwell. ${ }^{3}$

\section{Strengths and weaknesses of this study}

This review is the first to systematically focus on recruitment strategies in the UHA setting. With a reported growing number of UHAs presenting great challenges to modern-day healthcare provision, the conduct of RCTs in the UHA will inevitably develop as an area of research.

The review may be limited through its reliance on a single search of the ORRCA database, conducted in
February 2016, at which point the database contained publications relevant to recruitment published up to the end of December 2014. The ORRCA database continues to evolve as updates encompass newly published recruitment research. Updates to the database may have generated further UHA research articles since the search for this review. It is possible that any new update could make an important and significant contribution to this field because so little has been done in this area thus far.

The review may be limited because a single researcher reviewed the majority of the ORRCA entries, and it is possible that ORRCA may not have included all relevant articles in the first place. This work may also be at risk of publication bias, as we chose to exclude abstracts based on the assumption that these were unlikely to include all the data items we were interested in. Excluding abstracts may have resulted in omission of some potentially valuable information. Although some authors recommend the inclusion of conference abstracts within a review, ${ }^{64}{ }^{65}$ there is some evidence to suggest that there is discordance between the content of abstracts and the subsequent full-text publication, and as such, including abstracts may introduce unreliable data. ${ }^{66}{ }^{67}$ Another weakness is that the majority of recruitment studies were retrospective analyses of processes and events that occurred during the host RCT. These data were not necessarily collected with the intention of evaluating RCT recruitment strategies. This may limit the quality of the data and the use of the recommendations arising from the included studies.

Finally, a risk of bias assessment of the included recruitment studies was not performed because only one of the recruitment studies was an RCT (ie, a randomised, controlled evaluation of a recruitment intervention).

\section{Unanswered questions and future research}

Some articles within this review demonstrated inconsistent conclusions about the value of SDMs, who these should be and how these should operate. Future research should examine these issues in more depth, in a variety of clinical contexts, focusing on the roles of SDMs in different RCTs.

It is uncertain how well the results of studies using hypothetical RCTs can be translated to the conduct of real RCTs. There may be some validity in the findings from hypothetical RCTs, as highlighted in this review by the similarity of the results from studies that used real RCTs and hypothetical RCTs. Further work is required to ascertain the extent to which findings from studies that use hypothetical RCTs influence the design and conduct of real RCTs.

As part of this review, a classification system was devised in order to group together similarly designed recruitment research studies. Before any such classification system could be used more widely, it would require validation by testing its applicability to at least one further set of 
recruitment papers, preferably in a different healthcare setting.

This review highlighted strategies to deal with patients in the prehospital setting who required urgent treatment. However, not all patients who are cared for in the prehospital setting require treatment immediately. Some may require transport to hospital for further assessment and potential treatment. Further research could explore whether providing early trial information to such patients, based on their symptoms or presumed diagnosis, could affect trial recruitment should treatment be required later. This strategy could prove to be useful in a broader range of unscheduled hospital admissions.

Although some recommendations for optimising recruitment could be drawn from this review, the overall lack of research in this area, particularly among highquality, methodologically robust studies, is a limiting factor. Future recruitment studies in this clinical setting should focus on studies with higher methodological rigour, by developing novel interventions to optimise recruitment and prospectively evaluating their effectiveness through an appropriate study design.

\section{CONCLUSION}

There is a relative paucity of high-quality research on strategies to optimise recruitment to RCTs involving unscheduled hospital admissions. Some emerging recommendations include optimising information provision about the trial in the prehospital setting to improve recruitment where treatment is required urgently, or using technology to facilitate recruitment across many hospital sites. Screening log data can also provide useful insight to specific barriers to recruitment. Future research in this setting should focus on conducting studies with higher methodological rigour, by developing interventions to optimise recruitment and prospectively evaluating their effectiveness.

Acknowledgements The following are gratefully acknowledged for their assistance and support with this work: Shaun Treweek, Anna Kearney, Nicola Harman.

Contributors CR, LR, JR and JMB designed the study and formulated the protocol. $\mathrm{CR}$ and $\mathrm{KF}$ performed all data extraction, which was reviewed and critically analysed by CR, LR and JMB. CG facilitated access to the ORRCA database and critically appraised the review. CR produced the first draft of the manuscript and LR and JMB made substantial contributions to the formulation of the final version. All authors read and approved the final manuscript.

Funding CR is supported by the Medical Research Council (MRC) Hub for Trials Methodology Research Network and the Collaboration and Innovation for Difficult Trials in Invasive Procedures (ConDuCT-II) Hub for Trials Methodology Research (MR/K025643/1). The funders had no role in the study design, data collection and analysis, decision to publish or preparation of the manuscript.

Competing interests None declared.

Patient consent Obtained.

Provenance and peer review Not commissioned; externally peer reviewed.

Data sharing statement As this is a review of published literature, there are no additional data available.

Open Access This is an Open Access article distributed in accordance with the terms of the Creative Commons Attribution (CC BY 4.0) license, which permits others to distribute, remix, adapt and build upon this work, for commercial use, provided the original work is properly cited. See: http://creativecommons.org/ licenses/by/4.0/

(c) Article author(s) (or their employer(s) unless otherwise stated in the text of the article) 2018. All rights reserved. No commercial use is permitted unless otherwise expressly granted.

\section{REFERENCES}

1. Tudur Smith $\mathrm{C}$, Hickey H, Clarke M, et al. The trials methodological research agenda: results from a priority setting exercise. Trials 2014;15:32.

2. Schandelmaier S, von Elm E, You JJ, et al. Premature discontinuation of randomized trials in critical and emergency care: a retrospective cohort study. Crit Care Med 2016;44:130-7.

3. Cofield SS, Conwit R, Barsan W, et al. Recruitment and retention of patients into emergency medicine clinical trials. Acad Emerg Med 2010;17:1104-12.

4. Boland J, Currow DC, Wilcock A, et al. A systematic review of strategies used to increase recruitment of people with cancer or organ failure into clinical trials: implications for palliative care research. J Pain Symptom Manage 2015;49:762-72.

5. Fayter D, McDaid C, Eastwood A. A systematic review highlights threats to validity in studies of barriers to cancer trial participation. $J$ Clin Epidemiol 2007;60:990.e1-e33.

6. Mc Daid C, Hodges Z, Fayter D, et al. Increasing participation of cancer patients in randomised controlled trials: a systematic review. Trials 2006;7:16.

7. Ngune I, Jiwa M, Dadich A, et al. Effective recruitment strategies in primary care research: a systematic review. Qual Prim Care 2012;20:115-23.

8. Auster J, Janda M. Recruiting older adults to health research studies: a systematic review. Australas J Ageing 2009;28:149-51.

9. Provencher V, Mortenson WB, Tanguay-Garneau L, et al. Challenges and strategies pertaining to recruitment and retention of frail elderly in research studies: a systematic review. Arch Gerontol Geriatr 2014;59:18-24.

10. Ibrahim S, Sidani S. Strategies to recruit minority persons: a systematic review. J Immigr Minor Health 2014;16:882-8.

11. Caldwell PH, Hamilton S, Tan A, et al. Strategies for increasing recruitment to randomised controlled trials: systematic review. PLoS Med 2010;7:e1000368.

12. Fletcher B, Gheorghe A, Moore D, et al. Improving the recruitment activity of clinicians in randomised controlled trials: a systematic review. BMJ Open 2012;2:e000496.

13. Huynh L, Johns B, Liu SH, et al. Cost-effectiveness of health research study participant recruitment strategies: a systematic review. Clin Trials 2014;11:576-83.

14. Mapstone J, Elbourne D, Roberts I. Strategies to improve recruitment to research studies. Cochrane Database Syst Rev 2007;2:Mr000013.

15. Raftery J, Bryant J, Powell J, et al. Payment to healthcare professionals for patient recruitment to trials: systematic review and qualitative study. Health Technol Assess 2008;12:1-128.

16. Treweek S, Mitchell E, Pitkethly M, et al. Strategies to improve recruitment to randomised controlled trials. Cochrane Database Syst Rev 2010;1:Mr000013.

17. Watson JM, Torgerson DJ. Increasing recruitment to randomised trials: a review of randomised controlled trials. BMC Med Res Methodol 2006;6:34.

18. Burns KE, Prats CJ, Maione M, et al. The experience of surrogate decision makers on being approached for consent for patient participation in research. A multicenter study. Ann Am Thorac Soc 2017;14:238-45.

19. Whitesides LW, Baren JM, Biros MH, et al. Impact of individual clinical outcomes on trial participants' perspectives on enrollment in emergency research without consent. Clin Trials 2017;14:180-6.

20. Fordyce CB, Roe MT, Dickert NW. Maximizing value and minimizing barriers: patient-centered community consultation for research in emergency settings. Clin Trials 2017;14:88-93.

21. Leira EC, Ahmed A, Lamb DL, et al. Extending acute trials to remote populations: a pilot study during interhospital helicopter transfer. Stroke 2009;40:895-901.

22. Beshansky JR, Sheehan PR, Klima KJ, et al. A community consultation survey to evaluate support for and success of the IMMEDIATE trial. Clin Trials 2014;11:178-86.

23. Chow E, Zuberi M, Seto R, et al. Using real-time alerts for clinical trials: identifying potential study subjects. Appl Clin Inform 2011;2:472-80. 
24. Shaw L, Price C, McLure S, et al. Paramedic Initiated Lisinopril For Acute Stroke Treatment (PIL-FAST): results from the pilot randomised controlled trial. Emerg Med J 2014;31:994-9.

25. Abramson NS, Safar P. Deferred consent: use in clinical resuscitation research. Brain Resuscitation Clinical Trial II Study Group. Ann Emerg Med 1990;19:781-4.

26. Adeoye O, Pancioli A, Khoury J, et al. Efficiency of enrollment in a successful phase II acute stroke clinical trial. J Stroke Cerebrovasc Dis 2012;21:667-72.

27. Agård A, Hermerén G, Herlitz J. Patients' experiences of intervention trials on the treatment of myocardial infarction: is it time to adjust the informed consent procedure to the patient's capacity? Heart $2001 ; 86: 632-7$

28. Annane $\mathrm{D}$, Outin $\mathrm{H}$, Fisch $\mathrm{C}$, et al. The effect of waiving consent on enrollment in a sepsis trial. Intensive Care Med 2004;30:321-4.

29. Bellomo R, Cass A, Cole L, et al. Screening and study enrolment in the Randomized Evaluation of Normal vs. Augmented Level (RENAL) Replacement Therapy Trial. Blood Purif 2009;27:199-205.

30. Burns KE, Zubrinich C, Tan W, et al. Research recruitment practices and critically ill patients. A multicenter, cross-sectional study (the Consent Study). Am J Respir Crit Care Med 2013;187:1212-8.

31. Chlan L, Guttormson J, Tracy MF, et al. Strategies for overcoming site and recruitment challenges in research studies based in intensive care units. Am J Crit Care 2009;18:410-7.

32. Collins JF. Data and safety monitoring board issues raised in the VA Status Epilepticus Study. Control Clin Trials 2003;24:71-7.

33. Cook D, Arabi Y, Ferguson N, et al. Physicians declining patient enrollment in a critical care trial: a case study in thromboprophylaxis. Intensive Care Med 2013;39:2115-25.

34. Costescu DJ, Cullimore AJ. Lessons learned from a resident-led clinical trial in obstetrics. Clin Trials 2013;10:612-6.

35. Crowley ST, Chertow GM, Vitale J, et al. Lessons for successful study enrollment from the Veterans Affairs/National Institutes of Health Acute Renal Failure Trial Network Study. Clin J Am Soc Nephrol 2008;3:955-61.

36. Elkins JS, Khatabi T, Fung L, et al. Recruiting subjects for acute stroke trials: a meta-analysis. Stroke 2006;37:123-8.

37. Flaherty ML, Karlawish J, Khoury JC, et al. How important is surrogate consent for stroke research? Neurology 2008;71:1566-71.

38. Foster D, Cook D, Granton J, et al. Use of a screen log to audit patient recruitment into multiple randomized trials in the intensive care unit. Canadian Critical Care Trials Group. Crit Care Med 2000;28:867-71.

39. Glassberg AE, Luce JM, Matthay MA. National Heart, Lung, and Blood Institute Clinical Trials Network. Reasons for nonenrollment in a clinical trial of acute lung injury. Chest 2008;134:719-23.

40. Kendall B, Städeli R, Schegg B, et al. Clinical Trial Educator program-a novel approach to accelerate enrollment in a phase III International Acute Coronary Syndrome Trial. Clin Trials 2012;9:358-66

41. Kenyon S, Dixon-Woods M, Jackson CJ, et al. Participating in a trial in a critical situation: a qualitative study in pregnancy. Qual Saf Health Care 2006;15:98-101.

42. Roozenbeek B, Maas Al, Marmarou A, et al. The influence of enrollment criteria on recruitment and outcome distribution in traumatic brain injury studies: results from the impact study. J Neurotrauma 2009;26:1069-75.

43. Schats R, Brilstra EH, Rinkel GJ, et al. Informed consent in trials for neurological emergencies: the example of subarachnoid haemorrhage. J Neurol Neurosurg Psychiatry 2003;74:988-91.

44. Smith OM, McDonald E, Zytaruk N, et al. Rates and determinants of informed consent: a case study of an international thromboprophylaxis trial. J Crit Care 2013;28:28-39.

45. Smyth RM, Jacoby A, Elbourne D. Deciding to join a perinatal randomised controlled trial: experiences and views of pregnant women enroled in the Magpie Trial. Midwifery 2012;28:e538-e545.

46. Williams BF, French JK, White HD. HERO-2 consent substudy investigators. Informed consent during the clinical emergency of acute myocardial infarction (HERO-2 consent substudy): a prospective observational study. Lancet 2003;361:918-22.
47. Yamal JM, Robertson CS, Rubin ML, et al. Enrollment of racially/ ethnically diverse participants in traumatic brain injury trials: effect of availability of exception from informed consent. Clin Trials 2014:11:187-94.

48. Yuval R, Halon DA, Merdler A, et al. Patient comprehension and reaction to participating in a double-blind randomized clinical trial (ISIS-4) in acute myocardial infarction. Arch Intern Med 2000;160:1142-6.

49. Abboud PA, Heard K, Al-Marshad AA, et al. What determines whether patients are willing to participate in resuscitation studies requiring exception from informed consent? $J$ Med Ethics 2006;32:468-72.

50. Ali K, Roffe C, Crome P. What patients want: consumer involvement in the design of a randomized controlled trial of routine oxygen supplementation after acute stroke. Stroke 2006;37:865-71.

51. Bulger EM, Schmidt TA, Cook AJ, et al. The random dialing survey as a tool for community consultation for research involving the emergency medicine exception from informed consent. Ann Emerg Med 2009;53:341-50.

52. Clark DJ, Kolias AG, Corteen EA, et al. Community consultation in emergency neurotrauma research: results from a pre-protocol survey. Acta Neurochir 2013;155:1329-34.

53. Del Giudice A, Plaum J, Maloney E, et al. Who will consent to emergency treatment trials for subarachnoid hemorrhage? Acad Emerg Med 2009;16:309-15.

54. Goldstein JN, Espinola JA, Fisher J, et al. Public opinion of a stroke clinical trial using exception from informed consent. Int J Emerg Med 2010;3:385-9.

55. Newman JT, Smart A, Reese TR, et al. Surrogate and patient discrepancy regarding consent for critical care research. Crit Care Med 2012;40:2590-4.

56. Scotton WJ, Kolias AG, Ban VS, et al. Community consultation in emergency neurosurgical research: lessons from a proposed trial for patients with chronic subdural haematomas. Br J Neurosurg 2013;27:590-4

57. Sims CA, Isserman JA, Holena D, et al. Exception from informed consent for emergency research: consulting the trauma community. $J$ Trauma Acute Care Surg 2013;74:157-66.

58. Snowdon C, Elbourne D, Forsey M, et al. Views of emergency research (VERA): a qualitative study of women and their partners' views of recruitment to trials in severe postpartum haemorrhage. Midwifery 2012;28:800-8.

59. Donovan JL, Rooshenas L, Jepson M, et al. Optimising recruitment and informed consent in randomised controlled trials: the development and implementation of the Quintet Recruitment Intervention (QRI). Trials 2016;17:283.

60. Elliott D, Husbands S, Hamdy FC, et al. Understanding and improving recruitment to randomised controlled trials: qualitative research approaches. Eur Urol 2017;72:789-98.

61. Authority NHR. Applying a proportionate approach to the process of seeking consent-HRA Guidance, 2017.

62. Roberts I, Prieto-Merino D, Shakur H, et al. Effect of consent rituals on mortality in emergency care research. Lancet 2011;377:1071-2.

63. Sahan KM, Channon KM, Choudhury RP, et al. Refining the enrolment process in emergency medicine research. Eur $J$ Cardiovasc Med 2016;4:506-10.

64. Hopewell S, McDonald S, Clarke M, et al. Grey literature in metaanalyses of randomized trials of health care interventions. Cochrane Database Syst Rev 2007;2:MR000010.

65. Ziai H, Zhang R, Chan AW, et al. Search for unpublished data by systematic reviewers: an audit. BMJ Open 2017;7:e017737.

66. Tam VC, Hotte SJ. Consistency of phase III clinical trial abstracts presented at an annual meeting of the American Society of Clinical Oncology compared with their subsequent full-text publications. $J$ Clin Oncol 2008;26:2205-11.

67. Toma M, McAlister FA, Bialy L, et al. Transition from meeting abstract to full-length journal article for randomized controlled trials. JAMA 2006;295:1281-7. 